CORRECTIONS

\title{
Sepsis in children
}

In this Clinical Review by Adrian Plunkett and Jeremy Tong (BMJ 2015;350:h3017, doi:10.1136/bmj.h3017), box 1 ("Typical or important pathogens in sepsis") has an error: the subheading "Mosquito-borne disease" should have been "Mosquito-borne disease and other tropical diseases" because Burkholderia pseudomallei (included in the list) is not transmitted via mosquitoes.

Cite this as: BMJ 2015;351:h3704

๑ BMJ Publishing Group Ltd 2015 